\title{
Numerical integrators based on the Magnus expansion for nonlinear dynamical systems
}

\author{
M. Hajiketabi ${ }^{\mathrm{a}, *}$, F. Casas ${ }^{\mathrm{b}, * *}$ \\ ${ }^{a}$ Department of Mathematics, Imam Khomeini International University, Ghazvin, \\ 34149-16818, Iran \\ ${ }^{\mathrm{b}}$ Departament de Matemàtiques and IMAC, Universitat Jaume I, 12071 Castellón, \\ Spain
}

\begin{abstract}
Explicit numerical integration algorithms up to order four based on the Magnus expansion for nonlinear non-autonomous ordinary differential equations are presented and tested on problems possessing qualitative (very often, geometric) features that is convenient to preserve under numerical discretization. The range of applications covers augmented dynamical systems, highly oscillatory problems and nonlinear non-autonomous partial differential equations of evolution previously discretized in space.
\end{abstract}

Key words: Magnus expansion, Lie-group methods, preserving properties, augmented systems, high oscillation, non-autonomous PDEs of evolution

\section{Introduction}

Differential equations of the form

$$
Y^{\prime}=A(t, Y) Y, \quad Y(0)=Y_{0}
$$

where both $Y$ and $A$ are $n \times n$ matrices, are relevant in applications, especially when they are formulated in a particular matrix Lie group $G$ and $A$ belongs to the corresponding Lie algebra $\mathfrak{g}$. In that case, it can be shown that the solution $Y(t)$ belongs to the given Lie group for any time $t \geq 0$ (if the equation satisfies

* Corresponding author. Email: mohammadhajiketabi@gmail.com

**Email: Fernando.Casas@uji.es 
the usual regularity requirements). Relevant examples include the special orthogonal group $G=\mathrm{SO}(n)$ in rigid body mechanics and the symplectic group $\operatorname{Sp}(n)$ in Hamiltonian dynamics (in which case $n$ is an even number) [1]. The fact that the evolution of $Y$ is formulated in a Lie algebra is the mathematical counterpart of certain symmetries the physical system presents. Examples in point are the symplectic character of the Hamiltonian flow and the unitarity of the evolution operator in quantum mechanics.

The linear case, i.e., when Eq. (1) reads

$$
Y^{\prime}=A(t) Y, \quad Y(0)=Y_{0},
$$

is also very relevant in applications, especially in problems related with the time-dependent Schrödinger equations, such as the average Hamiltonian theory and quantum computing. In these fields, the Magnus expansion plays a significant role, both as a theoretical tool and as a numerical integrator. In essence, the solution of Eq. (2) is expressed as the exponential of a certain infinite series

$$
Y(t)=\mathrm{e}^{\Omega(t)} Y_{0}, \quad \Omega(t)=\Omega_{1}(t)+\Omega_{2}(t)+\Omega_{3}(t)+\cdots
$$

each term containing iterated integrals of nested commutators of $A$ evaluated at different times. Thus, for the first terms one has

$$
\begin{aligned}
& \Omega_{1}(t)=\int_{0}^{t} A\left(t_{1}\right) d t_{1}, \\
& \Omega_{2}(t)=-\frac{1}{2} \int_{0}^{t}\left[\int_{0}^{t_{1}} A\left(t_{2}\right) d t_{2}, A\left(t_{1}\right)\right] d t_{1},
\end{aligned}
$$

where $[A, B] \equiv A B-B A$ denotes the usual commutator.

In the particular case where $A(t)$ is periodic in Eq. (2), i.e., when there exists some $T>0$ such that $A(t+T)=A(t)$, the so-called Floquet-Magnus expansion has been proposed in [2] and subsequently applied in [3] in the context of solid state nuclear magnetic resonance problems, also comparing this approach with previous treatments based on average Hamiltonian theory and static perturbation theory.

There are numerous problems where Eqs. (1) and (2) have to be solved numerically, but in such a way that the qualitative properties of the exact solution are also shared by the approximations thus constructed. In particular, if Eq. (2) describes a quantum system, it is required that any approximation to $Y(t)$ be unitary. Otherwise, the probability conservation property might be compromised. Numerical integrators providing approximate solutions to Eqs. (1) and (2) belonging to the same Lie group $G$ are called Lie-group methods, and they have received a great attention in the literature during the last two decades (see, e.g., $[1,4,5]$ and references therein). 
Another area where using numerical integration methods preserving the Lie group structure is of paramount importance is in the context of the augmented dynamical systems technique. This was first proposed by Liu in [6] to integrate numerically any system of $k$ differential equations $x^{\prime}=f(t, x), x \in \mathbb{R}^{k}$, and later applied to a variety of settings, ranging from stiff differential equations to boundary value problems and systems with constraints [7-11].

Essentially, the idea consists in integrating not only the state variables $x$, but also the norm $\|x\|$. To proceed, the $(k+1)$-dimensional vector $y=(x,\|x\|)^{T}$ is introduced, so that it obeys the differential equation

$$
y^{\prime}=\frac{d}{d t}\left(\begin{array}{c}
x \\
\|x\|
\end{array}\right)=\left(\begin{array}{cc}
O_{k \times k} & \frac{f(t, x)}{\|x\|} \\
\frac{f^{T}(t, x)}{\|x\|} & 0
\end{array}\right)\left(\begin{array}{c}
x \\
\|x\|
\end{array}\right) \equiv A(t, y) y .
$$

It is assumed that $f$ satisfies a Lipschitz condition and $\|x\|>0$, so that the system is well defined. Otherwise, a convenient reparameterization renders the problem into this form. Although the dimension has increased by one, the augmented dynamical system (5) has an inherent symmetry, namely $A \in$ $\mathfrak{s o}(k, 1)$, the Lie algebra of the Lorentz group $\mathrm{SO}(k, 1)$ formed by all matrices $X$ with unit determinant such that $X J X^{T}=J$, with

$$
J=\left(\begin{array}{cc}
I_{k} & O_{k \times 1} \\
O_{1 \times k} & -1
\end{array}\right)
$$

whereas $A$ verifies $A J+J A^{T}=0$. Notice that $y^{T} J y=x \cdot x-\|x\|^{2}=0$, and thus this cone condition has to be exactly preserved by any numerical integrator applied to Eq. (5) to guarantee consistency. In this way, it is argued in [6] that the so-called group preserving schemes (GPS) conserve the group structure (and thus the cone condition). In consequence, they have the same asymptotic behavior as the original continuous system and do not induce spurious solutions or ghost fixed points. The simplest method in this setting is a first-order Lie-Euler discretization, either based on the matrix exponential

$$
y_{n+1}=\exp \left(h A\left(t_{n}, y_{n}\right)\right) y_{n}
$$

or the Cayley transformation and/or Padé approximants $[1,6]$.

As mentioned before, in many situations one has to use numerical techniques to construct approximate solutions of Eqs. (1) and (2) on a given time interval in such a way that the approximations thus obtained still possess as many qualitative properties of the exact solution as possible. Lie-group methods lead to approximations $Y_{k} \approx Y\left(t_{k}\right)$ on a grid $t_{0}=0<t_{1}<\cdots<t_{n}$ still belonging to the Lie group $G$. By contrast, general-purpose integrators such as explicit Runge-Kutta or extrapolation methods, when applied to Eqs. (1) 
and (2), typically furnish accurate solutions that do not stay in $G$, and thus they do not reproduce the correct qualitative behavior of the system, especially for long time integrations.

There are several possible strategies to integrate numerically equation (1) in such a way that the numerical approximation still belongs to $G$. For example, in the Runge-Kutta-Munthe-Kaas (RKMK) class of methods the problem is lifted from $G$ to the underlying Lie algebra $\mathfrak{g}$ with the inverse exponential map, then the associated differential equation in $\mathfrak{g}$ is solved with a Runge-Kutta method and finally this solution is mapped back to $G[1,12,13]$.

In the linear case, numerical methods based on the Magnus expansion have shown to be more efficient than other standard integrators whereas preserving the Lie group structure of the problem [14,15]. Given the excellent behavior shown by these schemes, it is hardly surprising that several attempts to generalize them to the nonlinear problem (1) have been conducted in the past. Thus, in [16], schemes of order $p$ can be derived by applying the idea of collocation to the differential equation defined in $\mathfrak{g}$. It turns out, however, that the methods obtained are implicit, although by relaxing the collocation conditions it is still possible to get explicit methods of order up to three.

In contrast, the approach followed in [17] leads in a natural manner to explicit integrators based on the Magnus expansion which are expressed in terms of arbitrary quadrature rules. This feature allows for a great flexibility of the procedure, in the sense that many different classes of problems modelled by Eq. (1) can be addressed essentially within the same framework just by introducing different discretizations to the integrals appearing in the formalism. Thus, one could choose a Newton-Cotes quadrature rule when $A$ is only known at equispaced points [18], a Gaussian rule to rise the order with the minimum number of evaluations [18], a Filon-type quadrature for highly oscillatory problems [19], etc.

Whereas the Magnus expansion for the nonlinear system (1) was explicitly derived in [17] and some particular numerical integrators were presented, it is our belief that a more exhaustive analysis of the technique and in particular a sound assessment of the schemes is still necessary. Thus, in this paper we show how to adapt the technique to different types of systems, ranging from (non-)autonomous ordinary differential equations evolving in Lie groups to highly oscillatory problems to non-autonomous partial differential equations of evolution type discretized in space. In this respect, we also show how this adaptivity can be further extended by introducing variable step size techniques, thus increasing significantly the efficiency of the proposed algorithms. More specifically, (i) we show that the nonlinear Magnus expansion applied in combination with the augmented dynamical systems technique produces higher order GPS, (ii) we introduce two variants of the procedure to deal 
with highly oscillatory problems and (iii) we show that the technique is also able to produce accurate results when applied to reaction-diffusion equations previously discretized in space.

\section{A review of numerical integrators based on the Magnus expan- sion}

\subsection{Schemes of orders 2, 3 and 4}

The numerical methods we consider in this paper were first proposed in [17] and are based on a generalization of the Magnus expansion for the solution of Eq. (1). In particular, it is shown that $Y(t)=\exp (\Omega(t)) Y_{0}$, where successive approximations of the matrix function $\Omega(t)$ are obtained by iteration,

$$
\Omega^{[m]}(t)=\int_{0}^{t} \sum_{k=0}^{\infty} \frac{B_{k}}{k !} \operatorname{ad}_{\Omega^{[m-1]}(s)}^{k} A\left(s, \mathrm{e}^{\Omega^{[m-1]}(s)} Y_{0}\right) d s, \quad m \geq 1,
$$

taking $\Omega^{[0]}(t) \equiv 0$. In particular, it is shown that, if terms up to $k=m-2$ are only kept in Eq. (8), then

$$
\Omega^{[m]}(t)=\Omega(t)+\mathcal{O}\left(t^{m+1}\right) .
$$

Equivalently, the Taylor expansions of $Y^{[m]}(t)=\mathrm{e}^{\Omega^{[m]}(t)} Y_{0}$ and of the exact solution $Y(t)$ around $t=0$ are identical up to terms of order $t^{m}, m \geq 1$. Thus, an approximation of order four is achieved by computing up to $m=4$, etc. As a matter of fact, only appropriate estimates of the integrals are indeed necessary to get consistent approximations up to the order considered, as we will show in the sequel.

If instead of the matrix equation (1), one considers the differential equation

$$
y^{\prime}=A(t, y) y, \quad y(0)=y_{0}, \quad y, y_{0} \in \mathbb{R}^{d}
$$

the same procedure is obviously valid, except that now the solution is obtained as $y^{[m]}(t)=\mathrm{e}^{\Omega^{[m]}(t)} y_{0}$.

To construct a numerical integrator of order $p$ on a time interval $\left[t_{0}, t_{f}\right]$ for equation (10) one takes some grid $0=t_{0}<t_{1}<\cdots<t_{N}=t_{f}$ with associated time increments $h_{n}=t_{n+1}-t_{n}$ for $0 \leq n \leq N-1$, and then determines the recurrence

$$
y_{0}=y\left(t_{0}\right), \quad y_{n+1}=\exp \left(\Omega^{[p]}\left(t_{n}+h_{n}, t_{n}\right)\right) y_{n} \approx y\left(t_{n+1}\right),
$$


where the dependence of $\Omega^{[p]}(t)$ also on the initial time has been made explicit. In doing so, it is sufficient to approximate in a consistent way all the integrals appearing in $\Omega^{[1]}, \Omega^{[2]}, \ldots$ up to the order considered and finally to compute the action of $\exp \left(\Omega^{[k]}\right)$ on vectors (if one is considering equation (10)) or matrices (for equation (1)). Special techniques (Chebyshev polynomials, Krylov methods, etc.) exist for the second case requiring typically less computational effort than the evaluation of the matrix exponential itself $([20,21]$ and references therein).

Thus, in particular, we consider the following schemes order 2, 3 and 4 .

Order two. According with the previous comments, only the first two iterations in Eq. (8) are required:

$$
\begin{aligned}
& \Omega^{[1]}\left(t_{n+1}, t_{n}\right)=\int_{t_{n}}^{t_{n}+h_{n}} A\left(s, y_{n}\right) d s, \\
& \Omega^{[2]}\left(t_{n+1}, t_{n}\right)=\int_{t_{n}}^{t_{n}+h_{n}} A\left(s, \mathrm{e}^{\Omega^{[1]}\left(s, t_{n}\right)} y_{n}\right) d s .
\end{aligned}
$$

If (as it is often the case), $\Omega^{[1]}$ can be evaluated exactly, and the trapezoidal rule is applied to $\Omega^{[2]}$, one has

$$
\begin{aligned}
& u=\Omega^{[1]}\left(t_{n+1}, t_{n}\right) \\
& v=\frac{h_{n}}{2}\left(A\left(t_{n}, y_{n}\right)+A\left(t_{n+1}, \mathrm{e}^{u} y_{n}\right)\right) \\
& y_{n+1}=\mathrm{e}^{v} y_{n}
\end{aligned}
$$

If this is not possible, we can always take the approximation $u=h_{n} A\left(t_{n}, y_{n}\right)$ in Eq. (13). This scheme will be referred to in the sequel as M2.

Order three. Several schemes are possible depending on the particular quadrature rule used to approximate the integrals in $\Omega^{[1]}, \Omega^{[2]}$ and $\Omega^{[3]}$. We have taken the 2-points Gauss-Legendre quadrature and the Simpson rule [18]. Since methods based on the later provide better results for the examples considered, we collect it here (M3 scheme):

$$
\begin{aligned}
Q_{1} & =\Omega^{[1]}\left(t_{n+1}, t_{n}\right) \\
Q_{2} & =h_{n} A\left(t_{n}+\frac{1}{2} h_{n}, \mathrm{e}^{\frac{1}{2} Q_{1}} y_{n}\right)-Q_{1} \\
u_{3} & =\frac{1}{2} Q_{1}+\frac{1}{4} Q_{2} \\
u_{4} & =Q_{1}+Q_{2} \\
Q_{3} & =-u_{4}+h_{n} A\left(t_{n}+\frac{1}{2} h_{n}, \mathrm{e}^{u_{3}} y_{n}\right)
\end{aligned}
$$




$$
\begin{aligned}
& Q_{4}=-u_{4}-Q_{2}+h_{n} A\left(t_{n}+h_{n}, \mathrm{e}^{u_{4}} y_{n}\right) \\
& u_{5}=u_{4}+\frac{2}{3} Q_{3}+\frac{1}{6} Q_{4}-\frac{1}{6}\left[Q_{1}, Q_{2}\right] \\
& y_{n+1}=\mathrm{e}^{u_{5}} y_{n}
\end{aligned}
$$

Order four. The same quadratures can be used to achieve order four by considering now up to $\Omega^{[4]}$ in Eq. (8). If the Simpson rule is applied one ends up with exactly the same sequence as in Eq. (14) with the additional computations

$$
\begin{aligned}
& u_{6}=u_{3}+\frac{1}{3} Q_{3}-\frac{1}{24} Q_{4}-\frac{1}{48}\left[Q_{1}, Q_{2}\right] \\
& Q_{5}=-u_{4}+h_{n} A\left(t_{n}+\frac{1}{2} h_{n}, \mathrm{e}^{u_{6}} y_{n}\right) \\
& Q_{6}=-u_{4}-Q_{2}+h_{n} A\left(t_{n}+h_{n}, \mathrm{e}^{u_{5}} y_{n}\right) \\
& v=u_{4}+\frac{2}{3} Q_{5}+\frac{1}{6} Q_{6}-\frac{1}{6}\left[Q_{1}, Q_{2}-Q_{3}+Q_{5}+\frac{1}{2} Q_{6}\right] \\
& y_{n+1}=\mathrm{e}^{v} y_{n}
\end{aligned}
$$

This feature opens a new possibility, namely the straightforward implementation of a variable step size strategy. Notice that, in the 4th-order scheme, one determines two numerical solutions at $t_{n+1}$ of orders three and four, respectively:

$$
\hat{y}_{n+1}=\mathrm{e}^{u_{5}} y_{n} \quad \text { and } \quad y_{n+1}=\mathrm{e}^{v} y_{n} .
$$

Then the quantity

$$
E_{r}=\left\|y_{n+1}-\hat{y}_{n+1}\right\|
$$

is used to estimate the local error. If $E_{r}$ computed at $t_{n+1}$ is below some prescribed tolerance tol, then the step from $t_{n}$ to $t_{n+1}$ is accepted and then we proceed to compute the approximation to the solution at $t_{n+2}$. If $E_{r}>$ tol instead, then the approximation at $t_{n+1}$ is rejected and a smaller step is chosen to compute a new approximation at $t_{n+1}$. In either case, it turns out that the new step size can be taken as [22]

$$
h_{\text {new }}=s h_{c}\left(\frac{\text { tol }}{E_{r}}\right)^{1 / 4}
$$

where $h_{c}$ denotes the current value of the step size and $s$ is a "safety factor" (typically, a number close to 1 , i.e., 0.8 or 0.9 ) chosen to decrease the probability of a rejection at the next step. With this error estimate and the resulting new step size, one typically advances with the higher-order result $y_{n+1}$ [23]. Moreover, in our case the estimate $E_{r}$ is obtained for free, since $\hat{y}_{n+1}$ has to be computed anyway as an intermediate stage in the calculation of $y_{n+1}$. The resulting scheme is called $\mathbf{M} 4$. 
In practice, we scale the $i$-th component of $y_{n+1}-\hat{y}_{n+1}$ by a factor $d_{i}=\left|\left(y_{n+1}\right)_{i}\right|$ to work with relative errors and additional parameters are introduced in order not to increase nor to decrease the step size too fast [23]. As for the initial step size, a possible choice is just $h_{0}=$ tol $/ 2$.

It is worth remarking that, although Magnus integrators have been originally devised for explicitly time dependent problems, it is worth noticing that the previous algorithms also provide numerical approximations in the autonomous setting, i.e., for nonlinear equations of the form

$$
y^{\prime}=A(y) y, \quad y(0)=y_{0} .
$$

This is in contrast with the linear case, since in that case the Magnus expansion just reproduces the exact solution.

\subsection{Numerical examples}

To illustrate the main features of the previous schemes, we consider next two simple examples.

Example 1. The motion of a free rigid body is described by the standard Euler equations [24]:

$$
y^{\prime}=\left(\begin{array}{l}
\Pi_{1}^{\prime} \\
\Pi_{2}^{\prime} \\
\Pi_{3}^{\prime}
\end{array}\right)=\left(\begin{array}{ccc}
0 & \frac{\Pi_{3}}{I_{3}} & \frac{-\Pi_{2}}{I_{2}} \\
\frac{-\Pi_{3}}{I_{3}} & 0 & \frac{\Pi_{1}}{I_{1}} \\
\frac{\Pi_{2}}{I_{2}} & \frac{-\Pi_{1}}{I_{1}} & 0
\end{array}\right)\left(\begin{array}{l}
\Pi_{1} \\
\Pi_{2} \\
\Pi_{3}
\end{array}\right)=A(y) y
$$

where $I_{1}, I_{2}, I_{3}>0$ are the three principal moments of inertia of the body, and $\boldsymbol{\Pi}=\left(\Pi_{1}, \Pi_{2}, \Pi_{3}\right)$ is the angular momentum in the moving frame. As is well known, this system possesses two quadratic invariants, namely

$$
C=\frac{1}{2}\|\mathbf{\Pi}\|^{2}
$$

the length of the constant angular momentum in the orthogonal body frame, and

$$
H=\frac{1}{2}\left(\frac{\Pi_{1}^{2}}{I_{1}}+\frac{\Pi_{2}^{2}}{I_{2}}+\frac{\Pi_{3}^{2}}{I_{3}}\right),
$$

the energy of the system. When the previous schemes based on the Magnus expansion are applied to Eq. (20), $C$ is exactly preserved by construction (up to round-off), whereas keeping track of the error in $H$ along the integration may help to give an assessment on the performance of the different integrators. 
For our experiment we take $I_{1}=3, I_{2}=2, I_{1}=3 / 2$, initial conditions $\Pi(0)=(1,1,1)$ and integrate until $t_{f}=100$ with different Magnus methods and different step sizes and/or tolerances. Then we compute the relative error in energy at $t_{f}$ and plot this error in terms of the required computational cost. This can be estimated as the total number of evaluations of the matrix $A$ plus the number of exponentials multiplied by vectors and the commutators required by each method, according with the following table:

\begin{tabular}{|c|c||c|c|c|}
\hline Method & Order & $A$ eval & Exp-vec & Comm \\
\hline \hline M2 & 2 & 2 & 2 & 0 \\
M3 & 3 & 4 & 4 & 1 \\
M4 & 4 & 6 & 6 & 2 \\
\hline
\end{tabular}

Thus, if $C_{\mathrm{M} x}$ denotes the computational cost of a given method based on Magnus, $\mathbf{M x}$, then $C_{\mathbf{M} 3} \sim 2.1 C_{\mathbf{M} 2}$, whereas $C_{\mathbf{M} 4} \sim 3.2 C_{\mathbf{M} 2}$. We collect in Figure 1 the results achieved by the previous schemes M2, M3 and M4. For comparison, we also include the result obtained by the standard explicit Runge-Kutta method of order 4 (Rk4).

Several comments are in order here. First, M2 exhibits for this example a third order of consistency and is in fact more efficient than M3. Second, M4 is the most efficient integrator within this family in a wide range of accuracies although its computational cost per step is the highest of all the methods considered. Third, all the schemes based on Magnus preserve the invariant Eq. (21) with 14-15 digits independently of the step size. This in contrast with other standard integrators, such as explicit Runge-Kutta methods. Although in this case there is no an explicit time dependence, and thus the schemes based on Magnus are not particularly appropriate, $\mathbf{M} 4$ still shows an efficiency comparable to $\mathbf{R k} \mathbf{4}$ for high accuracies.

One could think that our estimate of the computational cost of the different methods is rather crude. It nevertheless captures quite accurately the relative performance of the schemes: if one considers instead the CPU time required by each integration, a similar relative configuration of the respective curves is obtained.

Example 2. The next example is designed to illustrate the use of the Magnus integrators in conjunction with the augmented dynamical systems technique. To this end, we take the equation [25]:

$$
\begin{array}{ll}
x_{1}^{\prime}=x_{2}, & x_{1}(1)=0 \\
x_{2}^{\prime}=-x_{1}-x_{2}^{2}+\ln (t), & x_{2}(1)=1,
\end{array}
$$




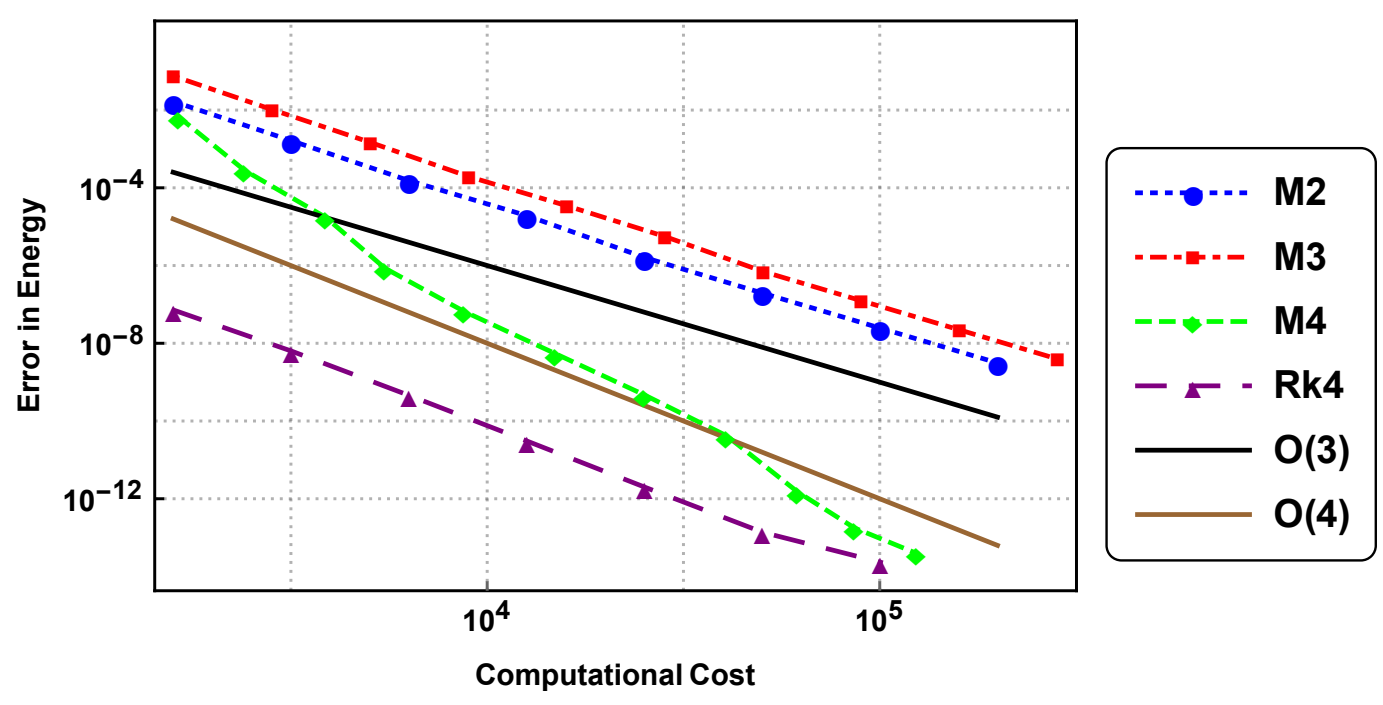

Fig. 1. Relative error in energy for the free rigid body obtained with different Magnus integrators and the explicit Runge-Kutta method of order 4 vs. their computational cost. Lines showing order 3 and 4 are also provided for comparison.

with exact solution

$$
x_{e}(t) \equiv\left(x_{1}(t), x_{2}(t)\right)^{T}=\left(\ln (t), \frac{1}{t}\right)^{T} .
$$

Here $f(t, x)=\left(x_{2},-x_{1}-x_{2}^{2}+\ln (t)\right)^{T}$, and so we apply Magnus methods to equation (5), where now $A$ is a $3 \times 3$ matrix. Of course, for solving this particular problem other methods can be more efficient, but we stress that our purpose here is to show that the Magnus integrators can be successfully applied in augmented dynamical systems.

We compute the numerical solution in the interval $t \in[1,101]$ with the first order scheme (7) and methods M2, M3 and M4, then determine the error as the Euclidean norm of the difference between $x_{e}$ and the numerical solution at $t_{f}=101$ for several step sizes and finally we plot this error as a function of the computational cost of each algorithm estimated as in Example 1. Thus we get Figure 2. It clearly illustrates the advantage of using a higher order integration scheme in problems where Liu's approach is applied: they not only preserve the inherent symmetry of the augmented dynamical system, but they do so with a much reduced computational cost for a given accuracy. We see then that, by applying Magnus expansion on augmented systems results in higher order GPS. These schemes could be applied, e.g., to get higher order approximations in combination with radial basis functions (RBFs) for PDEs $[26,27]$.

It is worth noticing that M2, M3 and M4 have essentially the same computational cost as the first-order scheme (7), but greater accuracy is always achieved. So we recommend replacing Eq. (7) by M2, M3 or M4 whenever 


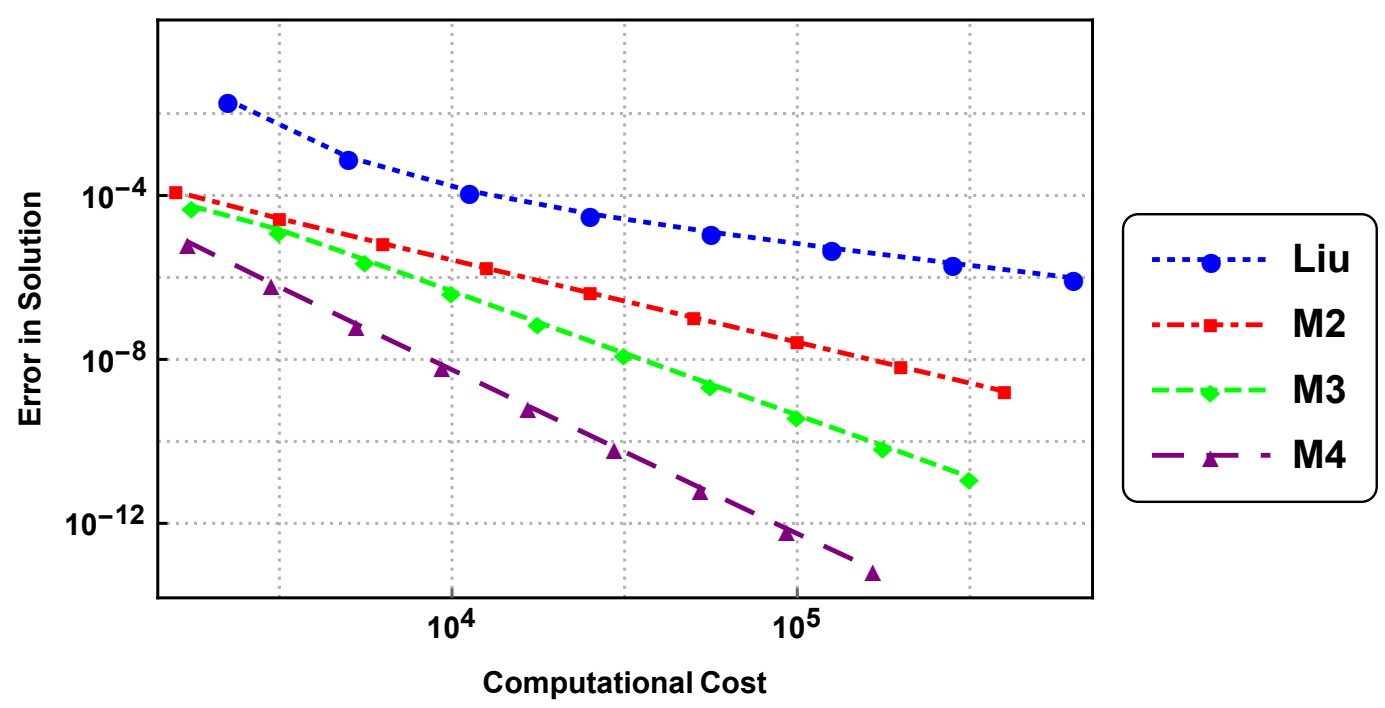

Fig. 2. Euclidean norm on the error in the solution for Example 2 obtained with Magnus integrators M2, M3 and M4 vs. their computational cost in comparison with Liu's approach.

the augmented dynamical system technique is employed.

\section{Generalization to highly oscillation problems}

During the last decade so much effort has been devoted to the design and analysis of numerical integrators especially adapted to solve different classes of ordinary differential equations exhibiting high oscillation. For example, we can cite trigonometric integrators and their modifications for solving the highly oscillatory second order autonomous system $q^{\prime \prime}=-A q+g(q)$ with a symmetric semi-definite matrix $A[28,29]$, adiabatic integrators for linear systems with time-dependent skew-Hermitian matrices [4], heterogeneous multiscale methods for mechanical systems subjected to fast vibrations [30], etc., for which special analytical techniques have been developed, such as the modulated Fourier expansions [4] and the averaging based on word series [31]. In all cases, special integrators are constructed (or adapted) to particular classes of problems exhibiting high oscillation.

There are, however, highly oscillatory time-dependent systems which are not easily formulated in such a way that these special integrators can be advantageously applied, but still can be described by Eq. (10). This is the case, in particular, of classical mechanical systems with time-dependent frequencies or nonlinear equations of the form $y^{\prime}=\varepsilon^{-1} A(t, y) y$, where $\varepsilon$ is a small parameter. For these systems we show next two different procedures to get numerical approximations based on the Magnus expansion depending on the the particular matrix $A(t, y)$. 
First approach. The first technique constitutes a generalization of the so-called modified Magnus methods proposed by Iserles [32,33] and further extended in [17] to the scalar equation $y^{\prime \prime}+a\left(t, y, y^{\prime}\right) y=0$. Here we consider a generic problem (10) with a high oscillatory $n \times n$ matrix $A$.

To begin with, assume that $y_{n} \approx y\left(t_{n}\right)$ has been computed and the goal is to determine the approximation $y_{n+1}$ at $t_{n+1}=t_{n}+h_{n}$. We first factorize the solution at intermediate times as

$$
y\left(t_{n}+\tau\right)=\mathrm{e}^{\tau A\left(t_{n}, y_{n}\right)} z(\tau), \quad \tau \geq 0 .
$$

By inserting Eq. (25) into $y^{\prime}=A(t, y) y$, we obtain the differential equation satisfied by $z(\tau)$, namely

$$
\frac{d z}{d \tau}=B(\tau, z) z, \quad z(0)=y_{n}
$$

where

$$
B(\tau, z)=\mathrm{e}^{-\tau A\left(t_{n}, y_{n}\right)}\left(A\left(t_{n}+\tau, \mathrm{e}^{\tau A\left(t_{n}, y_{n}\right)} z\right)-A\left(t_{n}, y_{n}\right)\right) \mathrm{e}^{\tau A\left(t_{n}, y_{n}\right)} .
$$

The new variable $z(\tau)$ may be seen thus as a correction to the approximation provided by the first term of the Magnus expansion discretized with Euler's method, and satisfies a differential equation of the same type as $y(t)$, but with some important differences: (i) $\|B\| \ll\|A\|$ by construction, and (ii) their entries are highly oscillatory since they are obtained from the entries of $\exp (\tau A)$. Therefore, if the Magnus expansion is applied to Eq. (26), approximations with a significantly smaller error are expected, even if the same quadratures are used.

To be more specific, consider the first Magnus iteration applied to Eq. (26) with a fixed step size $h$, and denote for simplicity $\Omega^{[1]}(h) \equiv \Omega^{[1]}\left(t_{n+1}, t_{n}\right)$,

$$
\Omega^{[1]}(h)=\int_{0}^{h} G_{1}(\tau) d \tau
$$

where

$$
G_{1}(\tau)=\mathrm{e}^{-\tau A\left(t_{n}, y_{n}\right)}\left(A\left(t_{n}+\tau, \mathrm{e}^{\tau A\left(t_{n}, y_{n}\right)} y_{n}\right)-A\left(t_{n}, y_{n}\right)\right) \mathrm{e}^{\tau A\left(t_{n}, y_{n}\right)},
$$

and discretize this integral with the Simpson rule, so that

$$
\Omega^{[1]}(h) \approx u(h) \equiv \frac{h}{6}\left(4 G_{1}\left(\frac{h}{2}\right)+G_{1}(h)\right),
$$

since $G_{1}(0)=0$. The resulting scheme

$$
y_{n+1}=\mathrm{e}^{h A\left(t_{n}, y_{n}\right)} \mathrm{e}^{u(h)} y_{n},
$$




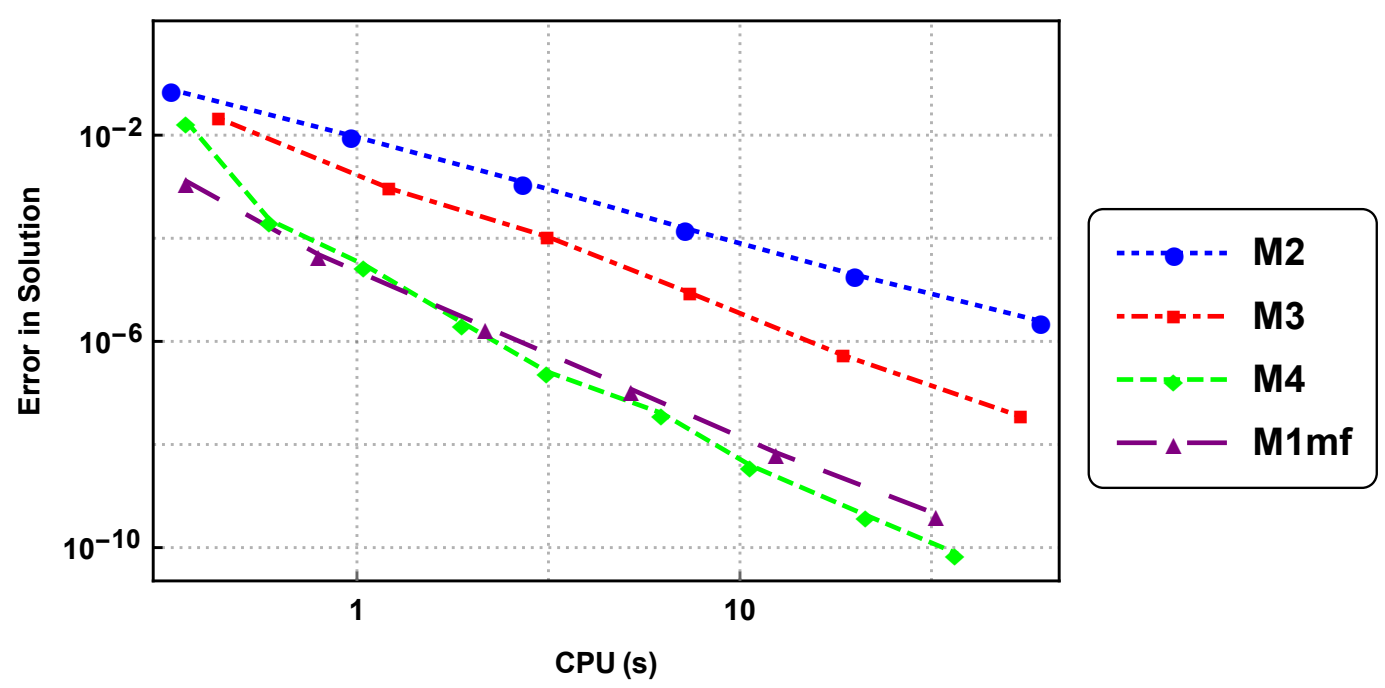

Fig. 3. Error in the solution for the highly oscillatory problem of Example 3. M1mf corresponds to the modified scheme (31).

although simple, already provides a reasonable description of the system, as is illustrated by the following example.

Example 3. It is defined by

$$
\begin{aligned}
& x_{1}^{\prime}=x_{2} \\
& x_{2}^{\prime}=-t x_{1}^{3}+g(t)
\end{aligned}
$$

with $\left.g(t)=t \cos \left(t^{2}\right)\left(-4 t+\cos ^{2}\left(t^{2}\right)\right)-2 \sin \left(t^{2}\right)\right)$. The function $g$ is chosen so that the exact solution when $x_{1}(0)=1, x_{2}(0)=0$ reads

$$
x_{1}(t)=\cos \left(t^{2}\right), \quad x_{2}(t)=-2 t \sin \left(t^{2}\right),
$$

clearly exhibiting an increasingly oscillatory character as time goes on. System (32) can be recast in the form Eq. (10) by introducing the vector $y=$ $\left(x_{1}, x_{2}, x_{3}=1\right)^{T}$ and the augmented matrix

$$
A(t, y)=\left(\begin{array}{ccc}
0 & 1 & 0 \\
-t x_{1}^{2} & 0 & g(t) \\
0 & 0 & 0
\end{array}\right)
$$

We integrate this problem in the interval $t \in[0,20]$ with the modified scheme (31) (which we call $\mathbf{M} 1 \mathbf{m f}$ ), M2, M3 and $\mathbf{M} 4$, determining the relative error in the solution as a function of the CPU time needed for the computation. The results are depicted in Figure 3.

We see that the modified scheme (31) with a fixed step size provides a similar 
accuracy as the much more elaborated 4th-order method with variable step size implementation. Notice that (modified) trigonometric integrators cannot applied to this system in a straightforward way.

Second approach. In the special, but important, situation when $A(t, y)$ has a purely imaginary spectrum and can be diagonalized for fixed values $t$ and $y$, yet another specially adapted procedure can be applied. In that case we can write

$$
A\left(t_{n}, y_{n}\right)=V D V^{-1}, \quad \text { with } \quad D=\operatorname{diag}\left(i \lambda_{j}\right), \quad \lambda_{j} \in \mathbb{R},
$$

so that

$$
G_{1}(\tau)=V \mathrm{e}^{-\tau D} \widetilde{M}(\tau) \mathrm{e}^{\tau D} V^{-1}
$$

with

$$
\widetilde{M}(\tau)=V^{-1}\left(A\left(t_{n}+\tau, \mathrm{e}^{\tau A\left(t_{n}, y_{n}\right)} y_{n}\right)-A\left(t_{n}, y_{n}\right)\right) V .
$$

The first term of the Magnus expansion applied to Eq. (26) reads thus

$$
\Omega^{[1]}(h)=V \int_{0}^{h} \mathrm{e}^{-\tau D} \widetilde{M}(\tau) \mathrm{e}^{\tau D} d \tau V^{-1} .
$$

Notice that the entries of the matrix in the previous integral are given by

$$
\left(\mathrm{e}^{-\tau D} \widetilde{M}(\tau) \mathrm{e}^{\tau D}\right)_{k \ell}=\mathrm{e}^{i\left(\lambda_{\ell}-\lambda_{k}\right) \tau} \widetilde{M}_{k \ell}(\tau) .
$$

As before, the next stage in the procedure consists in applying a conveniently chosen quadrature rule to Eq. (38). Given the highly oscillatory character of Eq. (39), it seems appropriate to consider a Filon-type method [19]. Since the entries in Eq. (38) are of the form $\int_{0}^{h} g(s) \mathrm{e}^{i \omega s} d s$, we propose the quadrature rule

$$
\int_{0}^{h} g(\tau) \mathrm{e}^{i \omega \tau} d \tau \approx b_{1} g(0)+b_{2} g(h / 2)+b_{3} g(h),
$$

where the weights $b_{i}$ are determined in such a way that Eq. (40) is exact for $g(\tau)=1, \tau, \tau^{2}$ :

$$
\begin{aligned}
& b_{1}(\omega, h)=\frac{i}{\omega}+\frac{3+\mathrm{e}^{i \omega h}}{h \omega^{2}}-4 i \frac{1-\mathrm{e}^{i \omega h}}{h^{2} \omega^{3}} \\
& b_{2}(\omega, h)=-4 \frac{1+\mathrm{e}^{i \omega h}}{h \omega^{2}}+8 i \frac{1-\mathrm{e}^{i \omega h}}{h^{2} \omega^{3}} \\
& b_{3}(\omega, h)=-i \frac{\mathrm{e}^{i \omega h}}{\omega}+\frac{1+3 \mathrm{e}^{i \omega h}}{h \omega^{2}}-4 i \frac{1-\mathrm{e}^{i \omega h}}{h^{2} \omega^{3}} .
\end{aligned}
$$

Notice that

$$
\left(b_{1}, b_{2}, b_{3}\right) \rightarrow h\left(\frac{1}{6}, \frac{2}{3}, \frac{1}{6}\right) \quad \text { when } \quad \omega \rightarrow 0
$$

and thus the Simpson rule is obtained in this limit. 


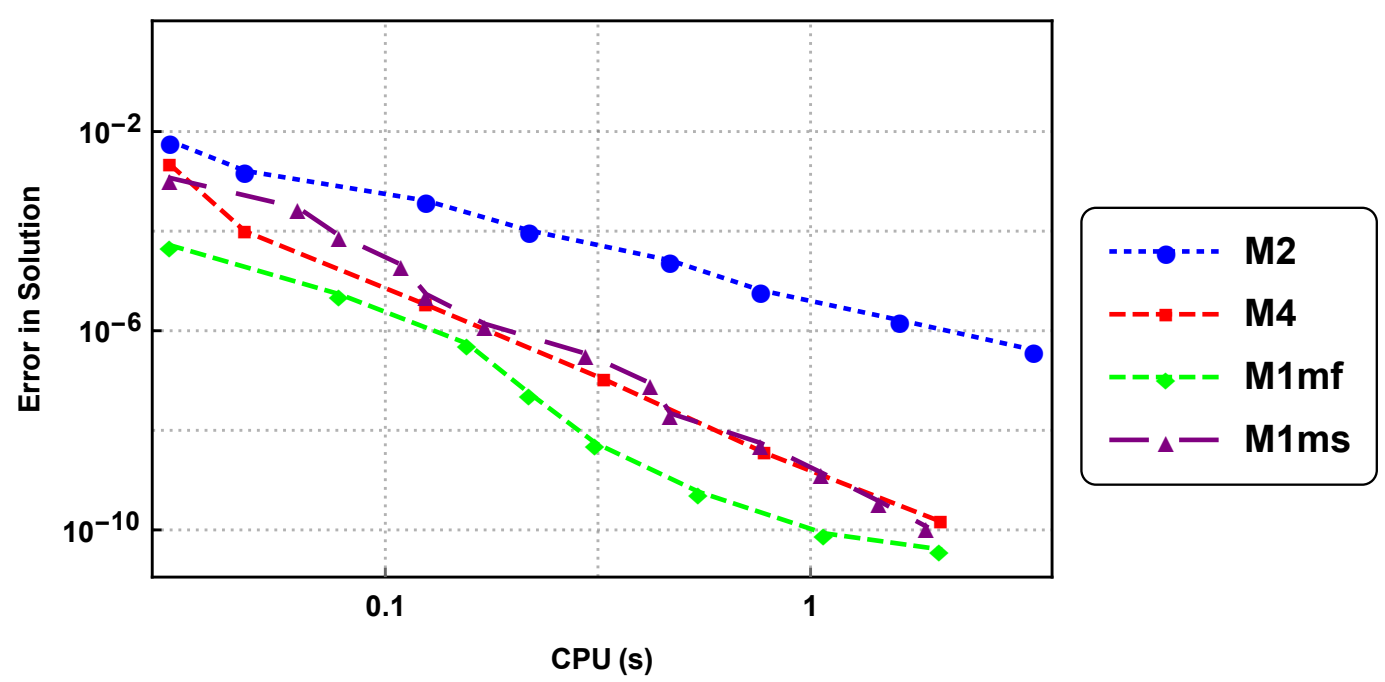

Fig. 4. Error in the solution for the highly oscillatory problem of Example 4. M1mf corresponds to the modified scheme (31) and M1ms corresponds to the modified scheme (43).

In our case, since $\widetilde{M}(0)=0$, the approximation reads

$$
\left(\Omega^{[1]}(h)\right)_{i j} \approx u(h) \equiv V_{i k}\left(b_{2}\left(\omega_{k \ell}, h\right) \widetilde{M}\left(\frac{h}{2}\right)+b_{3}\left(\omega_{k \ell}, h\right) \widetilde{M}(h)\right)_{k \ell}\left(V^{-1}\right)_{\ell j}
$$

where now $\omega_{k \ell} \equiv \lambda_{\ell}-\lambda_{k}$. Finally,

$$
y_{n+1}=\mathrm{e}^{h A\left(t_{n}, y_{n}\right)} \mathrm{e}^{u(h)} y_{n} .
$$

Example 4. With

$$
D=i\left(\begin{array}{cc}
1 & 0 \\
0 & \left(1+t\left(y_{1}+y_{2}\right)\right)^{-1}
\end{array}\right), \quad V=\left(\begin{array}{rr}
1 & 1 \\
1 & -1
\end{array}\right)
$$

we fix $x_{1}(0)=1, x_{2}(0)=0$ and compute the norm of error in $t=20$. As the exact solution we take, for convenience, the output generated by the function NDSolve of Mathematica with a very stringent tolerance, although any other procedure with the same requirements could aslo be used (e.g., the DOPR853 routine designed by Hairer and Wanner [23]). We can then apply Eq. (43) but also the first approach (31). The corresponding results are collected in Figure 4. We observe that the procedure (31) (line M1mf) gives the best results, whereas Eq. (43) (line M1ms) is as efficient as the much elaborated scheme M4. Notice that both M1mf and M1ms do not require the computation of commutators, and thus both are particularly favorable when the dimension of the problem is not so small. 


\section{Application to non-autonomous partial differential equations of evolution type}

The Magnus expansion can also be applied for the integration in time of certain linear time-dependent partial differential equations (PDEs) of evolution. A common approach in this setting is to first discretize in space, for which procedures such as finite differences, pseudo-spectral methods based on collocation with trigonometric polynomials, etc. exist. The resulting (large) system matrix of linear ordinary differential equation inherits fundamental properties of the corresponding PDE. For instance, if the original PDE corresponds to the Schrödinger equation of quantum mechanics, then the coefficient matrix of the resulting ordinary differential equation is skew-Hermitian. Although interpolatory Magnus integrators present some drawbacks in this setting due to the presence of commutators, it is possible to introduce some modifications and design efficient new classes of commutator-free schemes [34].

Motivated by the results achieved for the linear case, we try to generalize the treatment to reaction-diffusion equations. These appear in models describing the time evolution of chemical or biological species in a medium such as water or air. Another well-known example is the evolution of the the population of one or several species distributed in space under the action of two concurrent phenomena: reaction between species and diffusion which makes the species spread out in space. From a mathematical point of view, they belong to the class of semi-linear parabolic partial differential equations of the form

$$
\frac{\partial u}{\partial t}=C \Delta u+F(u, t)
$$

where each component of $u(x, t) \in \mathbb{R}^{d}$ represents the concentration (or the population) of one species, $C$ is a diagonal matrix containing the diffusion rates and $F$ models local interactions between species. The case $F(u, t)=r(t) u(1-$ u) corresponds to Fisher's (also called Kolmogorov-Petrovsky-Piskunov) equation, and is used to describe the spreading of biological populations.

For simplicity, we only consider one species and one space dimension, i.e.,

$$
\frac{\partial u}{\partial t}=\frac{\partial^{2} u}{\partial x^{2}}+r(t) u(1-u), \quad u(x, 0)=u_{0}(x),
$$

with periodic boundary conditions in the space domain $[0,1]$. We analyze, in particular, a periodic time dependence in the reaction term,

$$
r(t)=\beta \cos (\omega t), \quad \beta, \omega>0
$$

and initial condition $u_{0}(x)=\sin (2 \pi x)$. After discretization in space with finite 
differences we arrive at the differential equation

$$
\frac{d U}{d t}=A(U, t) \equiv D U+F(U, t)
$$

where $U=\left(u_{1}, \ldots, u_{N}\right)^{T} \in \mathbb{R}^{N}$, the second derivative has been approximated by the matrix $D$ of size $N \times N$ given by

$$
D=(\Delta x)^{-2}\left(\begin{array}{cccccc}
-2 & 1 & & & & 1 \\
1 & -2 & 1 & & \\
& 1 & -2 & 1 & \\
& & \ddots & \ddots & \ddots \\
1 & & & & 1 & -2
\end{array}\right)
$$

and $F(U, t)$ is now defined by

$$
F(U, t)=\beta \cos (\omega t)\left(u_{1}\left(1-u_{1}\right), \ldots, u_{N}\left(1-u_{N}\right)\right)
$$

We choose $N=100, \beta=\omega=1$ and compute the relative error (in the 2norm) at the final time $t=1$ by applying the previous algorithms based on the Magnus expansion, in particular schemes M2 and M4. We also compare with the well known 2nd-order Strang splitting scheme. This requires taking $t$ as a new coordinate $x_{t}$ and consider instead the enlarged system

$$
\frac{d}{d t}\left(\begin{array}{c}
U \\
x_{t}
\end{array}\right)=\left(\begin{array}{c}
D U \\
1
\end{array}\right)+\left(\begin{array}{c}
F\left(U, x_{t}\right) \\
0
\end{array}\right)
$$

The solution of each subsystem at time $t=h$ is given by

$$
\varphi_{h}^{[1]}:\left\{\begin{array}{l}
U(h)=\mathrm{e}^{h D} U_{0} \\
x_{t}(h)=h+x_{t}(0)
\end{array}, \text { and } \varphi_{h}^{[2]}:\left\{\begin{array}{c}
u_{1}(h)=\frac{\mathrm{e}^{h c} u_{1}(0)}{1+u_{1}(0)\left(\mathrm{e}^{h c}-1\right)} \\
\vdots \\
u_{N}(h)=\frac{\mathrm{e}^{h c} u_{N}(0)}{1+u_{N}(0)\left(\mathrm{e}^{h c}-1\right)} \\
x_{t}(h)=c x_{t}(0)
\end{array}\right.\right.
$$

respectively, with $c \equiv \beta \cos \left(\omega x_{t}(0)\right)$. The Strang splitting applied to this system

$$
\psi_{h}=\varphi_{h / 2}^{[1]} \circ \varphi_{h}^{[2]} \circ \varphi_{h / 2}^{[1]},
$$

results in the following algorithm for the step $t_{n} \mapsto t_{n+1}$, providing an approx- 


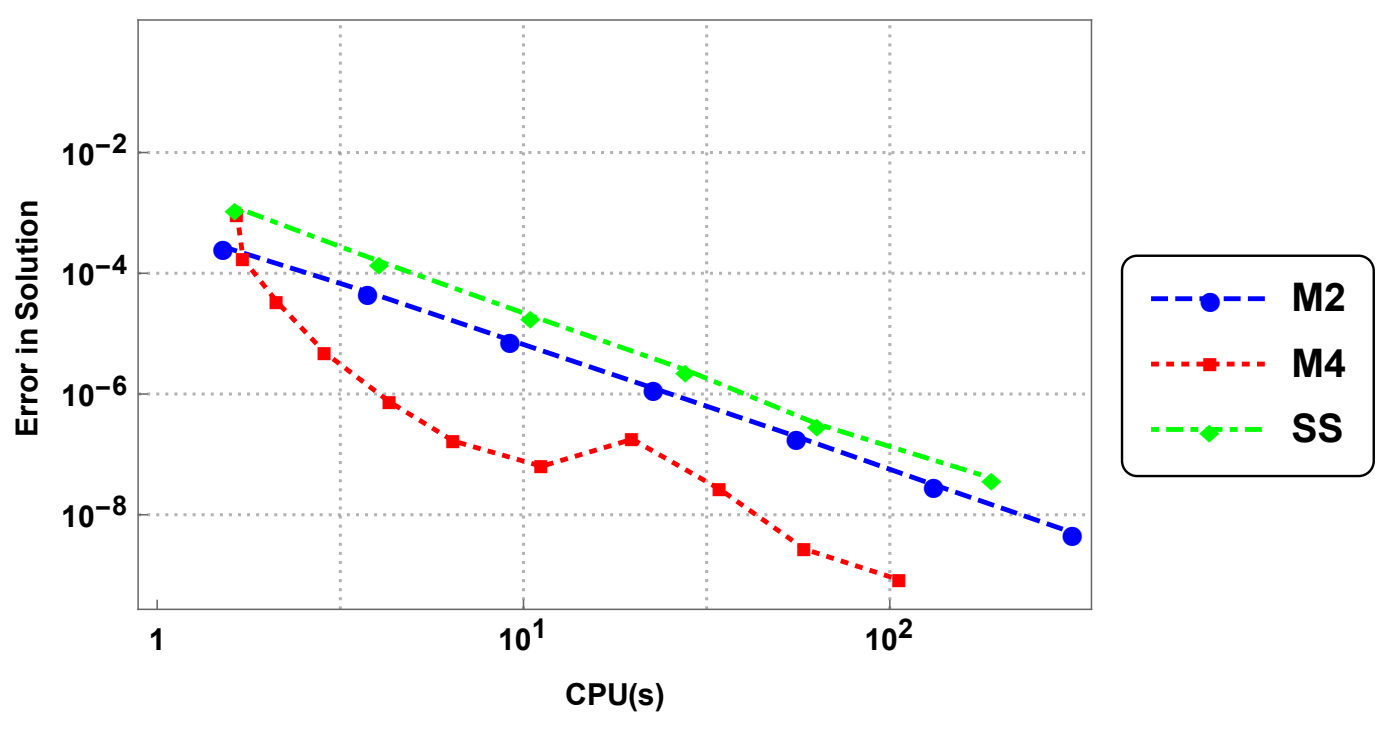

Fig. 5. Relative error in the solution for the Fisher problem, equation (46). SS corresponds to the Strang splitting (54).

imation $U_{n+1} \approx U\left(t_{n}+h\right)$ :

$$
\begin{aligned}
& V_{1 / 2}=\mathrm{e}^{\frac{h}{2} D} U_{n} \\
& c=\beta \cos \left(\omega\left(t_{n}+h / 2\right)\right) \\
& \operatorname{do} j=1, \ldots, N \\
& \quad V_{1, j}=\frac{\mathrm{e}^{h c} V_{1 / 2, j}}{1+V_{1 / 2, j}\left(\mathrm{e}^{h c}-1\right)}
\end{aligned}
$$

end do

$$
U_{n+1}=\mathrm{e}^{\frac{h}{2} D} V_{1}
$$

where $V_{i}=\left(V_{i, 1}, \ldots, V_{i, N}\right)^{T}, i=\frac{1}{2}, 1$.

Here again we compute the reference solution by applying the function NDSolve of Mathematica with a very stringent tolerance. The corresponding results are depicted in Figure 5. Notice that the scheme M4 is more efficient that the Strang splitting SS. It is worth noticing that, although one could construct higher order methods by composing the 2nd-order Strang splitting, the resulting schemes involve necessarily some negative coefficients, and thus present severe instabilities. Only splitting or composition methods with complex coefficients are appropriate [5]. This is not the case of methods based on the Magnus expansion. 


\section{Concluding remarks}

Nonlinear differential equations of the form Eq. (1) possessing specific qualitative properties appear frequently in applications. Very often these properties are associated with a special structure of the matrix $A(t, y)$, namely $A$ belongs to some Lie algebra. In particular, if $A$ is skew-symmetric, then the norm of the vector solution $y(t)$ is preserved along the evolution, a feature that seems convenient to retain also when the differential equation is approximately solved by numerical schemes. In contrast with other numerical integrators like explicit Runge-Kutta and multi-step methods, the explicit schemes based on the Magnus expansion considered here do preserve by construction these features, thus providing the corresponding approximations a qualitatively superior performance. The methods are formulated as the exponential of a conveniently chosen approximation of an integral involving the matrix $A$ evaluated at different times and commutators for order higher than two.

Although the methods are originally designed to deal with the explicitly time-dependent nonlinear equation (1), they prove to be also competitive in the treatment of autonomous problems with exactly the same formulation, as shown by the examples considered here. This is an attractive feature of the schemes in comparison with other structure-preserving integrators such as splitting methods: although in principle they can be applied to nonautonomous problems, removing previously the time dependency by considering time as a new coordinate is typically required, and this may deteriorate their overall efficiency [4].

Using the Magnus expansion in combination with the augmented dynamical systems technique produces higher order GPS, so that we can apply the variable-step methods presented here instead of the basic first-order GPS for the same family of problems. In addition, highly oscillatory problems formulated as Eq. (1) constitute a natural area of application of these schemes. With just a previous linear transformation, M1 already provides good results for general matrices $A$. If $A$, on the other hand, has a purely imaginary spectrum and can be diagonalized, the formalism can be adapted and special quadrature rules can be implemented.

Magnus-based schemes can also be applied to the time integration of reactiondiffusion equations previously discretized in space. We have illustrated this feature by applying several Magnus integrators to the well known Fisher equation and we plan to extend the treatment to other classes of explicitly timedependent nonlinear PDEs, such as the Gross-Pitaevskii equation. In that case, however, and as in linear problems, the presence of commutators in the algorithms might be problematic, and so one possibility might be to generalize the treatment carried out in [34] to the nonlinear setting. 
Analytic approximations based on the Fer expansion have also been considered in the linear case (Eq. (2) and applied to different physical problems [15,35]. In contrast to the Magnus expansion, the Fer approach consists in writing the solution of Eq. (2 as an infinite product of exponentials of matrices, each one containing higher order corrections. It is even possible to construct numerical integrators by truncating appropriately the infinite series appearing in each exponential, and replacing the integrals by suitable quadratures, just as in the case of Magnus integrators [36]. It is then natural to consider their generalization to nonlinear problems, just as we have carried out here for the Magnus expansion, and this may constitute an interesting line of research to pursue.

Acknowledgements

MH acknowledges the Iran Ministry of Science for financial support while on leave at the Institut de Matemàtiques i Aplicacions de Castelló, Universitat Jaume I (Spain). FC has been supported by Ministerio de Economía, Industria y Competitividad (Spain) through the project MTM2016-77660-P (AEI/FEDER, UE).

\section{References}

[1] A. Iserles, H.Z. Munthe-Kaas, S.P. Nørsett, and A. Zanna. Lie-group methods. Acta Numerica, 9:215-365, 2000.

[2] F. Casas, J. A. Oteo, and J. Ros. Floquet theory: exponential perturbative treatment. J. Phys. A: Math. Gen., 34:3379-3388, 2001.

[3] E. S. Mananga and T. Charpentier. Introduction of the Floquet-Magnus expansion in solid-state Nuclear Magnetic Resonance spectroscopy. J. Chem. Phys., 135:044109, 2011.

[4] E. Hairer, Ch. Lubich, and G. Wanner. Geometric Numerical Integration. Structure-Preserving Algorithms for Ordinary Differential Equations. SpringerVerlag, Second edition, 2006.

[5] S. Blanes and F. Casas. A Concise Introduction to Geometric Numerical Integration. CRC Press, 2016.

[6] C.-S. Liu. Cone of non-linear dynamical system and group preserving schemes. Int. J. Non-Linear Mech., 36:1047-1068, 2001.

[7] Abbasbandy, R.A. Van Gorder, M. Hajiketabi, and M. Mesrizadeh. Existence and numerical solution of periodic traveling wave solutions to the Casimir equation for the Ito system. Commun. Nonlinear Sci. Numer. Simulat., 27:254262,2015 . 
[8] S. Abbasbandy, R.A. Van Gorder, and M. Hajiketabi. The Lie-group shooting method for radial symmetric solutions of the Yamabe equation. CMES-Comp. Model. Eng., 104:329-351, 2015.

[9] C.-S. Liu. Nonstandard group-preserving schemes for very stiff ordinary differential equations. CMES-Comp. Model. Eng., 9:225-272, 2005.

[10] C.-S. Liu. A new sliding control strategy for nonlinear system solved by the Liegroup differential algebraic equation method. Commun. Nonlinear Sci. Numer. Simulat., 19:2012-2038, 2014.

[11] C.-S. Liu. A novel Lie-group theory and complexity of nonlinear dynamical systems. Commun. Nonlinear Sci. Numer. Simulat., 20:39-58, 2015.

[12] H. Munthe-Kaas. Runge-Kutta methods on Lie groups. BIT, 38(1):92-111, 1998.

[13] H. Munthe-Kaas. High order Runge-Kutta methods on manifolds. Appl. Numer. Math., 29:115-127, 1999.

[14] A. Iserles and S.P. Nørsett. On the solution of linear differential equations in Lie groups. Phil. Trans. Royal Soc. A, 357:983-1019, 1999.

[15] S. Blanes, F. Casas, J.A. Oteo, and J. Ros. The Magnus expansion and some of its applications. Phys. Rep., 470:151-238, 2009.

[16] A. Zanna. Collocation and relaxed collocation for the Fer and the Magnus expansions. SIAM J. Numer. Anal., 36(4):1145-1182, 1999.

[17] F. Casas and A. Iserles. Explicit Magnus expansions for nonlinear equations. J. Phys. A: Math. Gen., 39:5445-5461, 2006.

[18] L.M. Delves and J.L. Mohamed. Computational Methods for Integral Equations. Cambridge University Press, Second edition, 1992.

[19] A. Iserles and S. P. Nørsett. On quadrature methods for highly oscillatory integrals and thier implementation. BIT, 44:755-772, 2004.

[20] C. B. Moler and C. F. Van Loan. Nineteen dubious ways to compute the exponential of a matrix, twenty-five years later. SIAM Review, 45:3-49, 2003.

[21] C. Lubich. From Quantum to Classical Molecular Dynamics: Reduced Models and Numerical Analysis. European Mathematical Society, 2008.

[22] J.M. Sanz-Serna and M.P. Calvo. Numerical Hamiltonian Problems. Chapman \& Hall, 1994.

[23] E. Hairer, S.P. Nørsett, and G. Wanner. Solving Ordinary Differential Equations I, Nonstiff Problems. Springer-Verlag, Second revised edition, 1993.

[24] J. E. Marsden and T. S. Ratiu. Introduction to Mechanics and Symmetry. Springer-Verlag, 2nd edition, 1999.

[25] C.-S. Liu and W.-S. Jhao. The second Lie-group $\mathrm{SO}_{o}(n, 1)$ used to solve ordinary differential equations. J. Math. Research, 6:18-37, 2014. 
[26] M. Hajiketabi and S. Abbasbandy. The combination of meshless method based on radial basis functions with a geometric numerical integration method for solving partial differential equations: Application to the heat equation. Eng. Anal. Bound. Elem., 87:36-46, 2018.

[27] M. Hajiketabi, S. Abbasbandy, and F. Casas. The Lie-group method based on radial basis functions for solving nonlinear high dimensional generalized Benjamin-Bona-Mahony-Burgers equation in arbitrary domains. Appl. Math. Comput., 321:223-243, 2018.

[28] R.I. McLachlan and A. Stern. Modified trigonometric integrators. SIAM J. Numer. Anal., 52:1378-1397, 2014.

[29] B. Wang, A. Iserles, and X. Wu. Arbitrary-order trigonometric fourier collocation methods for multi-frequency oscillatory systems. Found. Comput. Math., 16:151-181, 2016.

[30] M.P. Calvo and J.M. Sanz-Serna. Heterogeneous multiscale methods for mechanical systems with vibrations. SIAM J. Sci. Comput., 32:2029-2046, 2010 .

[31] Ph. Chartier, A. Murua, and J.M. Sanz-Serna. Higher-order averaging, formal series and numerical integration I: B-series. Found. Comput. Math., 10:695-727, 2010 .

[32] A. Iserles. On the global error of discretization methods for highly-oscillatory ordinary differential equations. BIT, 42:561-599, 2002.

[33] A. Iserles. On the method of Neumann series for highly oscillatory equations. BIT, 44:473-488, 2004.

[34] S. Blanes, F. Casas, and M. Thalhammer. High-order commutator-free quasiMagnus exponential integrators for non-autonomous linear evolution equations. Comp. Phys. Commun., 220:243-262, 2017.

[35] E.S. Mananga and T. Charpentier. Revisiting the applications of FloquetMagnus and Fer expansion approaches in physics and solid-state NMR. Int. J. Modern Phys. B, 32:1850236, 2018.

[36] S. Blanes, F. Casas, and J. Ros. High order optimized geometric integrators for linear differential equations. BIT, 42:262-284, 2002. 